Presented at the 1977 Particle Accelerator Conference, Chicago, IL, March $16-18,1977$

\title{
A 3-DIMENSIONAL BEAM SCANNING SYSTEM FOR PARTICLE RADIATION THERAPY
}

Ch. Leemann, J. Alonso, H. Grunder, E. Hoyer,

G. Kalnins, D. Rondeau, J. Staples, and F. Voelker

\section{March 1977}

Prepared for the U. S. Energy Research and

Development Administration under Contract W-7405-ENG-48

\section{For Reference}

Not to be taken from this room

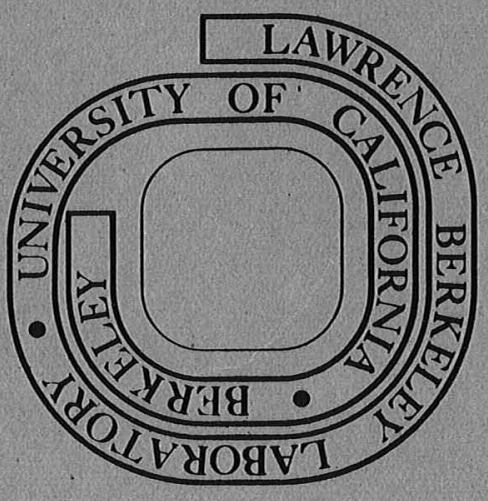




\section{LEGAL NOTICE}

This report was prepared as an account of work sponsored by the United States Government. Neither the United States nor the United States Energy Research and Development Administration, nor any of their employees, nor any of their contractors, subcontractors, or their employees, makes any warranty, express or implied, or assumes any legal liability or responsibility for the accuracy, completeness or usefulness of any information, apparatus, product or process disclosed, or represents that its use would not infringe privately owned rights. 
Ch. Leemann, J. Alonso, H. Grunder, E. Hoyer, G. Kalnins

D. Rondeau, J. Staples and F. Voelker

Lawrence Berkeley Laboratory

University of California

Berkeley, California 94720

\section{Summary}

In radiation therapy treatment volumes up to several liters have to be irradiated. Today's charged particle programs use ridge filters, scattering foils, occluding rings collimators and boluses to shape the dose distribution. ${ }^{l}$ An alternative approach, scanning of a small diameter beam, is analyzed and tentative systems specifications are derived. Critical components are scheduled for fabrication and testing at LBL.

\section{Introduction}

Apart from biological advantages whose detailed investigation constitutes at present a substantial research effort heavy charged particle beams have the physical advantage of spatially extremely well defined dose distributions. Their successful application in a wide range of therapeutic situations requires means to irradiate volumes as 1 arge as $30 \mathrm{~cm} \times 30 \mathrm{~cm} \times 15 \mathrm{~cm}$. Prescribed dose levels should be attained within a few precent, irradiation of healthy tissue minimized and the distortion of dose distributions caused by tissue heterogencities should be compensated for. The time for one treatment should not exceed a few minutes; jeally about 1 minute is aimed at. 3-dimensional scanning secms to meet these requirements. The basic components of a scamning system are two magnets sweeping the bcam over the treatment area, a device or procedure to change beam energy quickly, a device to control beall intensity and a conputer control system.

\section{Analysis of Procedure}

\section{Spatial Characteristics}

We briefly examine the relation between the prescribed dose distribution $D(X, Y, Z)$ and the sweep pattern. From this criteria for the step sizes will be derived. We assume that over the duration of one treatment $(\sim 60 \mathrm{~s})$ the detailed time structure of dose accumulation is bjologically immaterial. The scanning pattern is then described by a distribution function $I(X, Y, Z)$ :

$$
\int_{-\infty}^{x} \int_{-\infty}^{y} \int_{0}^{z} I\left(X_{0}, Y_{0}, Z_{0}\right) d X_{0} d Y_{0} d Z_{0}=N(X, Y, Z)
$$

where $N(X, Y, Z)$ is proportional to the total number of particles delivered with beam centroid positions $X_{0} \leqslant X, Y_{0} \leqslant Y$ and range $Z_{0} \leqslant Z_{0}$. We describe the contribution to the dose at $(X, Y, Z)$ from a beam with centroid coordinates $\left(X_{0}, Y_{0}\right)$ and range $Z_{0}$ by a function $g\left(X, X_{0}, Y, Y_{0}, Z, Z_{0}\right)$. The relation between $I$ and $D$ is then expressed as a Fredholu equation of the first

*This work wis done with support of the National Inst itute of (ancer of the Department of llealth, liducation and lielfare. kind:

$$
\begin{gathered}
D(X, Y, Z)=\int_{-\infty}^{+\infty} \int_{-\infty}^{+\infty} \int_{z 1}^{z 2} I\left(X_{0}, Y_{0}, z_{0}\right) g\left(X, X_{0}, Y, Y_{0}, Z, Z_{0}\right) \\
d X_{0} d Y_{0} d z_{0} .
\end{gathered}
$$

Exact solutions of this deconvolution problem will have to be found numerically and $g\left(X, X_{0}, Y, Y_{0}, Z, Z_{0}\right)$ has to be determined first by solving a "transport" or "Boltzmann" - type integro-differential equation.

To define systems parameters a simpler approach suffices. We assume a homogencous medium, neglect secondary beam components resulting from nuclear interactions and assume a beam profile indepedent of $z$. Then we obtain:

$g_{X}\left(X-X_{0}, Y-Y_{0}, Z-Z_{0}\right)=g_{X}\left(X-X_{0}\right) \cdot g_{y}\left(Y-Y_{0}\right) \cdot g_{z}\left(Z-Z_{0}\right)$.

This allows a simple solution of equation(2) in terins of Fourier transforms:

$\bar{D}(k, \ell, m)=\bar{I}(k, \ell, m) \cdot \bar{g}_{x}(k) \cdot \bar{g}_{x}(\ell) \cdot \bar{g}_{z}(m)$

where the bar denotes the Fourier transform. The (spatial) frequency range of $D$, and thercfore attainable resolution, is obviously limited by the finite beam spot size and longitudinal extent of the Bragg peak. Also a finite step scanning pattern, resulting in a $\delta$-function like $I(X, Y, Z)$ yiclds very smooth dose distributions (variations $<1 \%$ ) if the step sjze does not exceed $\sim$ one beam half-width. Lower limj.ts for beam spot sizes and therefore useful step sizes are determined by multiple scattering and are of the order of 2 to $3 \mathrm{~mm}$ for ${ }^{12} \mathrm{C}$ and 5 to $7 \mathrm{~mm}$ for protons for ranges in tissuc between $20 \mathrm{~cm}$ and $30 \mathrm{~cm}$.

Sweep Velocity Requirements

In the following required wagnet rise times are derived. We envisage a system with a fast scan in $X$-direction at constant $(Y, Z)$, a slower scan in the $Y$-direction and the slowest change in $Z$, i.e. energy. The treatment volume is thought to be subdivided in cubical elementary volumes or "cells" whose length $\mathrm{L}_{c}$ corresponds to the characteristic beam dimensions.

For a given treatment volume $V$, treatment time $T$ and macroscopic duty cycle $n$ of the accelerator we obtain for the scamning velocity in $x$-direction, $v_{x}$, and the corresponding time $\Delta t_{X}$ :

$v_{x}=(r, T)^{-1} V / L_{c}^{2} \quad$ (3) and $\Delta t_{x}=n^{\prime} I^{\prime} L_{x} I_{c}^{2} / V$.

There exists a minimum time $\Delta t_{x}$, min independent of treatment volune corresponding to $\mathrm{L}_{x, \min }$, the minimum 
length of the $X$-scan compatible with a given volume:

$$
\begin{aligned}
& \Delta t_{x, \min }=\eta_{T} L_{c}^{2} /\left(L_{y, \max } \cdot L_{g, \text { max }}\right) \\
& L_{x, \min }=V /\left(L_{y, \max } \cdot L_{s, \max }\right)
\end{aligned}
$$

If a finite-size step pattern is used an efficient system requires a sweep speed $v_{y}$ in $\mathrm{Y}$-direction

given by:

$v_{y}=k \cdot \frac{L_{c}}{\Delta t_{x} \text {, min }} \quad k \sim 5$

The time interval required to complete a 2-dimensional scan at a given energy is of the order $n T L_{c} / L_{z}$ and

energy changes must be performed in a fraction thereof or be synchronized with beam-off periods, e.g. between synchrotron pulses.

\section{Intensity Control}

In order to achieve the desired dose distribution energy deposition at each scan position has to be controlled by controlling beam intensity and/or adjusting the scanning speed. Our design uses a finite-step $X$-scan, accumulating dose at constant $X$, then stepping to the next position with a sweep speed of $100 \mathrm{nls}^{-1}$ for a $400 \mathrm{McV} / \mathrm{amu}$ Carbon beam. A fast (a few $\mu s$ ) beam switch allows the beam to be turned off during the step if required. The design sweep speed while stepping is 5 times greater than the value $V_{x}$ from equation (5) for $L_{c}=4 m n, ~ T T=30 s$ and $V=10$ liters. For a $30 \mathrm{~cm}$ scan this corresponds to going from $-B_{\max }$ to $+B_{\max }$ in $3 \mathrm{~ms}$.

\section{System Design}

\section{Scanning Magnets and Power Supplies}

Optimum magnet parameters depend on the properties of the overall beam delivery system in which they are incorporated. In horizontal beams they are easily incorporated in the final drift length which can be fairly long. In the vertical beams or in the isocentric systems considered for therapy overall economics may dictate a location further upstream. We estimate the power requixements for the first scanning magnet. The magnetic ficld is:

$B_{\text {max }}=\frac{\theta(B \rho)}{L_{m}}=\frac{L_{x}(B \rho)}{2 T_{12} \cdot L_{m}}$

where $\mathrm{T}$ is the transfer matrix from magnet center to target, $L_{m}$ the magnet length and $L_{x}$ the length of the scan. The stored energy is:

$u_{s}=4 \cdot \frac{10^{7}}{8 \pi} \alpha \frac{\varepsilon \sqrt{B_{X}} \sqrt{\beta}_{Y}}{L_{m}} \cdot\left(\frac{L_{X}}{2}\right)^{2} \frac{\left(B_{\rho}\right)^{2}}{T_{12}{ }^{2}}$

where $\pi \varepsilon_{x}=\pi \varepsilon_{y}=\pi \varepsilon$ is the beam emittance, $\beta_{x}, \beta_{y}$ are the betil functions at the magnet and the factor $\alpha$ takes into account that the actual magnetic field volume is somewhat larger than the beam aperture. For a spot size $X_{f}=\sqrt{\varepsilon} \sqrt{\beta}_{f}$ we obtain $\beta_{x}$, min $=T_{12}{ }^{2} / \beta_{f}$, $\beta_{y, \min }=\mathrm{T}_{34}{ }^{2} / \beta_{\mathrm{f}}$.
Equation (9) becomes:

$U_{s}=\alpha \frac{10^{7}}{2 \pi}: \frac{\varepsilon^{2}(B p)^{2}}{L_{m}}\left(\frac{L_{x}}{{ }_{x f}}\right)^{2} \cdot \frac{T_{34}}{T_{12}}$

For $\alpha=1.5, \varepsilon=10^{-5} \mathrm{~m}, x_{f}=3 \mathrm{~mm}, L_{x}=30 \mathrm{~cm}, L_{m}=1 \mathrm{~m}$

and $B \rho=6.5 \mathrm{~T}_{\mathrm{m}}$ we obtain:

$\mathrm{U}_{\mathrm{s}, \min }=25 \cdot \frac{\mathrm{T}_{34}}{\mathrm{~T}_{12}}(\mathrm{~J})$

and with a risetime of $1.5 \mathrm{~ms}\left(0\right.$ to $B_{\max }$ ) the required peak power is :

$\mathrm{P}_{\text {peak }} \simeq 35 \cdot \frac{\mathrm{T}_{34}}{\mathrm{~T}_{12}}$.

This approximate analysis of power requirements is valid if:

$\mathrm{T}_{12}^{2} \gg \frac{\mathrm{L}_{\mathrm{m}} \cdot \mathrm{L}_{\mathrm{x}} \cdot \mathrm{X}_{\mathrm{f}}}{4 \varepsilon}$

Also if $\mathrm{T}_{34}$ is very small it's maximum value over the magnet length must be used. At present a ferrite core scanning magnet is being built at LBL. Apertures are slightly larger than minimally required in order to have some flexibility in ion optical arrangements. Typical parameters are: $L_{m}=1 \mathrm{~m}$, aperture $=100 \mathrm{~mm}$ $x$ 40min, $B_{\max }=0.22 \mathrm{~T}$ and $\mathrm{U}_{\mathrm{s}}=100 \mathrm{~J}$.

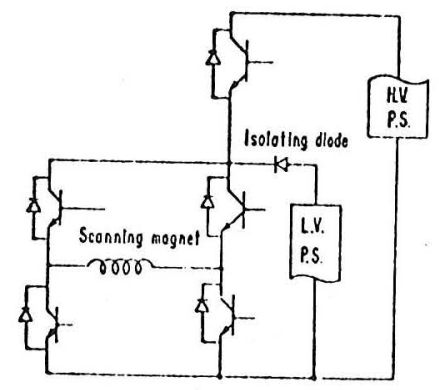

$X 8 L \geqslant 13-340$

Fig. 1 Fast scanning magnet Power supply schematic.

In all investigated layouts the stored energy in the second magnet is larger, $\sim 1.0$ to $\sim 3.5 \mathrm{~kJ}$, due to increased aperture requirements and this magnet is therefore the logical choice for the slow scan.

The fast magnet is powered by four transistor actuators in a bridge circuit allowing a constant current of either polarity by regulating the appropriate actuators. Power for the holding current is provided from a low voltage power supply connected through an isolating diode. A fifth actuator switches 
Fig. 2

Control System

Schematic.

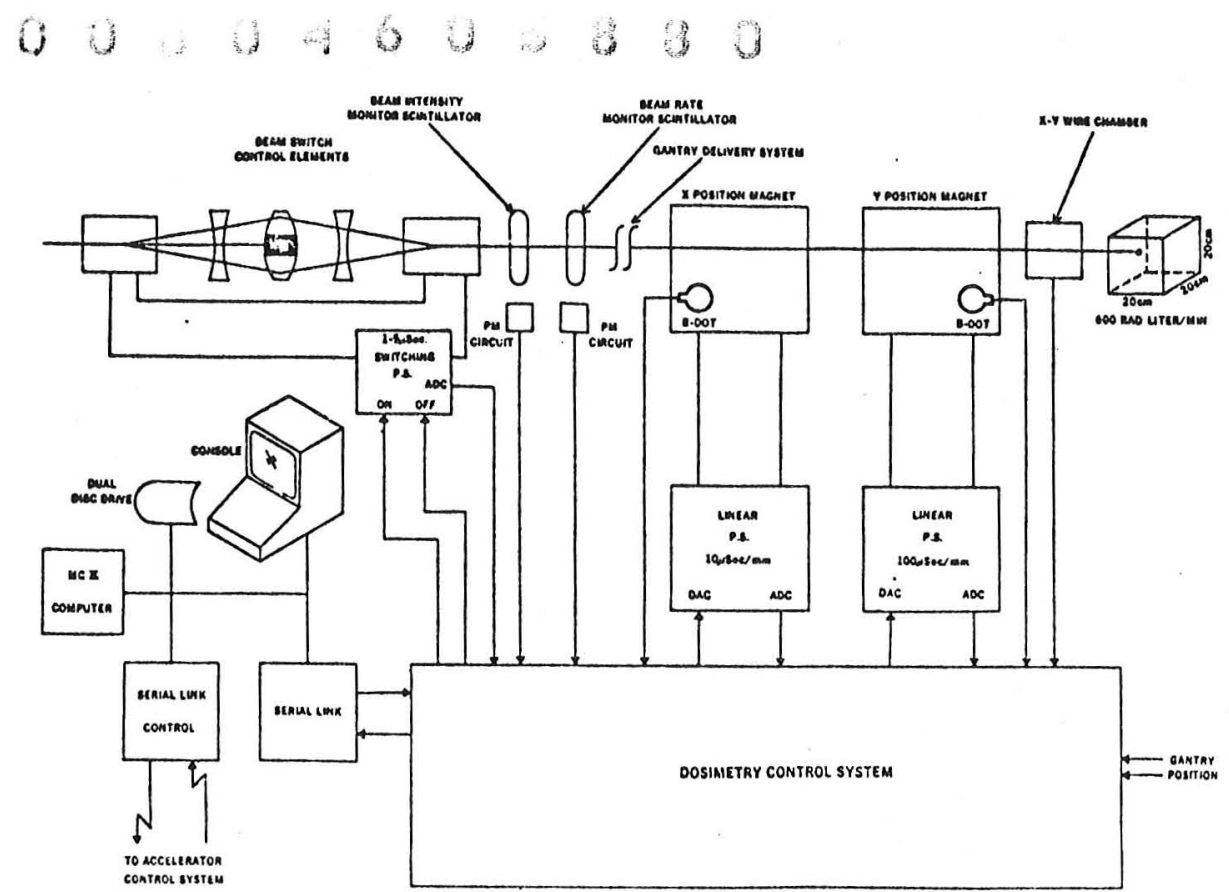

a $100 \mathrm{~V}$ power supply to the bridge and by turning on hard the appropriate actuators in the bridge the magnet is driven to the new holding current. The fifth actuator is switched off and the regulating mode resumes. (Fig. 1) Work on a test power supply has started.

For the slow nagnet an analogous approach substituting SCR's for the transitor actuators is planned.

\section{Beam on/off Switch}

The beam switch simple consists of a magnet capable of swceping the beam by one to two beam diameters over a matching collimator in a few $\mu s$. Preliminary designs cnvisage a 1 to $2 \mathrm{~m}$ long ferrite core, one turn magnet operating at fields of less than $10 \mathrm{~m} T$.

\section{Energy Modulation}

It scems entirely fcasible that a synchrotron designed for medical applications could perform energy changes on a pulse to pulse basis. In the interest of simple operation however, or for installation at existing facilities such as the LBL Bevalac, a variable thickness degrader appears more attractive. Such a degrader must be located after the last bean transport elements very close to the patient since it introduces a substantial increase in emittance. For protons, for example, even if a low $Z$ degrader such as $B e$ or $C$ is used, emittance in each plane increases by a factor of approximately 10 to 50 and an energy spread of a few $\mathrm{MeV}$ is introduced for the required range adjustments. A clevice similar to the LASL range shifter ${ }^{2}$ seems ideally suited.

\section{Monitoring and Computer Control}

Fig. (2) depicts schematically a tentative design of the control system. Two scintillator and photomultiplier circuits are used, one for monitoring dose accumulation and one for continuous rate monitoring allowing interruption of the process if the beam intensity exceeds as prẹset level. Scanning magnet fields are measured by $B$-coils with a multi-wire proportional chamber serving for position verification With only a few hundred $\mu$ s allotted for each scan position real time computer control will be difficult if a precision of a few percent is aimed at. Actual control and data acquistion will be performed in hardware (dosimetry control system) with the computer interacting on a plane to plane basis, i.e. when an energy change occurs. The computer, e.g. a ModComp MCII, will be equipped with a control console, a disk drive containing the dose profiles and two high speed serial links, one to the accelerator control computer and one to the dosimetry control system.

In a plane $z_{k}$ each cell is characterized by the set $\left(D_{t}, X_{i}, Y_{j}, B_{z}\right)$, where $D_{t}$ is the total specified particle count, $X_{i}, Y_{j}$ it's $(X, Y)$ - coordinates and $B_{z}$ a modifier used to calculate the cell memory address from the scanning magnet fields $B_{X}$, $B_{y}$ for each energy.

In operation the $z_{k}$, dose profile is downloaded in one half of the local memory in the dosimetry control system. The bean is positioned at $x_{k \max }$, $Y_{j \max }$ and the cell is exposed until the desired dose is obtained (or exceeded). Address verification and dose comparison is performed on the basis of a $1_{\mu s}$ cycle. Then the actual dose is recorded and the beam is moved to $X_{k m a x-1}$, etc. When all $X_{i}, Y_{j \max }$ are satisfied a step to $Y_{j m a x-1}$ occurs. Whife the plane $z_{k}$ is cxposed, actual recorded doses at $z_{k-1}$ are transmitted back to the disk and desired dose values for $z_{k+1}$ are loaded into the local memory.

\section{Conclusion}

It scems feasible to construct a beam delivery system which allows therapeutic irradiations to be performed with a precision limited only by the physical characteristics of particle beams and their interaction with matter. Critical subsystems, fast scanning magnet, power supply and parts of the control system are under construction at LBI.. A meaningful application of this technique requires of course diagnostic procedures of equal precision. Such techniques are becoming available however with 3 -dimensional reconstruction procedures (i.e. CAT scans) or the use of auto-radioactive beams. ${ }^{3}$

\section{References}

1. K. Crowe, et.a]., LBL-4235, 1975.

2. D. J. Liska, This Conference.

3. C. A. Tobias, et.a1., Phys. Lett. 37A, 2, 119-120 1971. 
This report was done with support from the United States Energy Research and Development Administration. Any conclusions or opinions expressed in this report represent solely those of the author(s) and not necessarily those of The Regents of the University of California, the Lawrence Berkeley Laboratory or the United States Energy Research and Development Administration. 